\title{
Study of factors affecting range of motion after total knee arthroplasty in Indian patients

\author{
Ravindra GR $^{1,{ }^{*}}$, Marthand Kulkarni ${ }^{2}$ and Sharan Patil ${ }^{3}$
}

\author{
${ }^{1}$ Department of Orthopaedic, Mahadevappa Rampure Medical College, Kalaburagi, Gulbarga, Karnataka-585105, India \\ ${ }^{2}$ Department of Orthopaedic, Khaja Bandanawaz Institute of Medical Sciences, Kalaburagi, Gulbarga, Karnataka-585104, India \\ ${ }^{3}$ Department of Orthopaedic, Sparsh Hospital, Yeshwanthpur, Bangalore, Karnataka-560022, India
}

\begin{abstract}
Background: Range of motion is an important indicator of the success of total knee arthroplasty. To our knowledge there are no studies assessing the factors affecting the range of motion after total knee arthroplasty on Indian population which prompted us to undertake this study.
\end{abstract}

Method: The present prospective study for all those patients with advanced arthritis of knee satisfying inclusion criteria, between August 2009 to June 2011. A total of 430 total knee replacements were done among 327 patients during this period out of which 354 primary total knee replacements among 254 patients were included in the study. Follow ups were done at 3 months, 6 months, one year and 2 years following procedure.

Result: The average age was 63.8 years with a range of $50-80$ years. $66.1 \%$ of the patients were female and $33.9 \%$ were male. The preoperative diagnosis was osteoarthritis in $81.1 \%$ of patients and $18.9 \%$ had rheumatoid arthritis. All poly implant was used in $83.1 \%$ of patients and in $16.9 \%$ of patients metal backed was used. The posterior tibial slope had no correlation with the postoperative range of motion at all the points of follow up. The preoperative range of motion had a statistical correlation with the postoperative range of motion at all the points of follow up and in all the age groups, $\mathrm{p}<0.0001$.

Conclusion: A more keen watch has to be kept in all young female patients with early onset of arthritis and mange them aggressively with conservative therapy.

Keywords: total knee arthroplasty; range of motion; modified Insall's knee society scoring system

*Correspondence author: Dr. Ravindra GR, Senior Resident, Department of Orthopaedic, Mahadevappa Rampure Medical College, Kalaburagi, Gulbarga, Karnataka-585105, India. Email: ravzhere@gmail.com

Received 4 January 2021; Revised 2 March 2021; Accepted 10 March 2021; Published 20 March 2021

Citation: Ravindra GR, Kulkarni M, Patil S. Study of factors affecting range of motion after total knee arthroplasty in Indian patients. J Med Sci Res. 2021; 9(2):56-63. DOI: http://dx.doi. org/10.17727/JMSR.2021/9-9

Copyright: (C) 2021 Ravindra GR et al. Published by KIMS Foundation and Research Center. This is an open-access article distributed under the terms of the Creative Commons Attribution License, which permits unrestricted use, distribution, and reproduction in any medium, provided the original author and source are credited. 


\section{Introduction}

In the past, severe degenerative disease of the knee was frequently treated by arthrodesis. Total knee replacements are now performed routinely all over the world. In 1826, Barton attempted one of the first simple resection of an ankylosed joint with some initial success [1].

Range of motion is an important indicator of the success of total knee arthroplasty. It has been found to be positively associated with knee function scores, walking ability and stair climbing activity. Increased motion is associated with improved function and increased patient satisfaction.

Several studies have demonstrated that patients require an average of 67 degree of flexion for the swing phase of gait, 83 degree to climb stairs, 90 degree to descend stairs, and 93 degree to rise from a seated position. Flexion greater than 105 degree is necessary for kneeling and squatting during activities of daily living and for religious acts [2]. Conventional designs were limited to ROM upto 90 degree [3].

A number of reports have described the factors affecting range of motion after total knee arthroplasty. Among the important factors are preoperative range of motion, tibiofemoral varus/ valgus angle, patients age, preoperative knee function score, and postoperative therapy $[1,4]$. The importance of other potential factors like posterior femoral condyle osteophyte, posterior tibial slope, soft tissue balancing is less well documented in the English literature $[5,6]$. To our knowledge there are no studies assessing the factors affecting the range of motion after total knee arthroplasty on Indian population which prompted us to undertake this study. The objectives of this study were to assess the various factors affecting the postoperative range of motion.

\section{Materials and methods}

The present prospective study for all those patients with advanced arthritis of knee satisfying inclusion criteria, who were treated at "Sparsh Hospital" Bangalore between August 2009 to June 2011. A total of 430 total knee replacements were done among 327 patients during this period out of which 354 primary total knee replacements among 254 patients were included in the study based on the inclusion and exclusion criteria. Follow ups were done at 3 months, 6 months, one year and 2 years following procedure.

Inclusion criteria: (1) Patients aged between 50 - 80 years, (2) Advanced arthritis.

Exclusion criteria: (1) Age more than 80 years and less than 50 years, (2) Uncontrolled diabetes and hypertensive with renal functional disturbances, Neuropathy, (3) Presence of systemic and local infections, (4) Revision total knee replacements, (5) Previous Osteotomies, (6) Postoperative infection, (7) Previous patellectomy, (8) Foreign patients.

\section{Method of collection of data}

Depending upon the age and medical fitness with their associated comorbidities, patients with bilateral advanced arthritis underwent total knee replacement in single anesthesia (bilateral single stage) or staggered with a gap of 2 to 7 days between surgeries or under two separate hospitalisation (bilateral staggered). Collection of data from patients who underwent total knee arthroplasty are such as, history by verbal communication, clinical examination both local and systemic, routine blood investigations as for any major surgery and ECG, 2D ECHO was done, special investigations like pulmonary function tests, dobutamine stress. ECHO was done in relevant cases, radiological examination, photographic documentation: Preoperative photographs and videos were recorded. Preoperative knee scores were calculated by using modified Insall's knee society scoring system [7], which includes both subjective and functional components. The nature of total knee replacement including its need, advantages, disadvantages, outcomes and consequences were explained to the patients and their attendants and a valid informed consent was taken. Pre-anesthetic evaluation and fitness was obtained before the surgery. Data was collected through pre-defined proforma.

Post-operative radiograph (Anteroposterior and lateral) view were taken to see the prosthetic positioning in both sagittal and coronal planes. The patients were advised to walk with walker support for 4 to 6 weeks. Follow up was done at three months, six months, one year and two years. Functional outcome was assessed using modified Insall's knee scoring system. 


\section{Statistical analysis}

Descriptive statistics such as range, frequencies, percentages, means, standard deviations were used to present the data. Correlation coefficients were calculated. Kruskul Wallis chi-square test was used to test the significance of difference between quantitative variables and Yate's chi square test for qualitative variables. A ' $p$ ' value less than 0.05 is taken to denote significant relationship. A correlation coefficient more than \pm 0.5 denotes association between the two variables. Data analysis was performed by using EPInfo and SPSS v20.0 software.

\section{Result}

The average age was 63.8 years with a range of 50 - 80 years with $50.8 \%$ of patient in $60-69$ year age group. $66.1 \%$ of the patients were female and $33.9 \%$ were male. The body mass index ranged from $17.6-$ 46.7 with $40.9 \%$ of patients between 25.1 - 30 group with a mean of 27.5 (Table 1).

Table 1: Baseline characteristics.

\begin{tabular}{|lcc|}
\hline \multicolumn{1}{|c}{ Characteristics } & Number & Percentage \\
\hline Age (years) & 70 & 27.6 \\
$50-59$ & 129 & 50.8 \\
$60-69$ & 55 & 21.7 \\
$70-80$ & & \\
Sex & 86 & 33.9 \\
Male & 168 & 66.1 \\
Female & & \\
Body mass index & 89 & 35 \\
Up to 25 & 104 & 40.9 \\
$25.1-30$ & 61 & 24.1 \\
Above 30 & & \\
\hline
\end{tabular}

The preoperative diagnosis was osteoarthritis in $81.1 \%$ of patients and $18.9 \%$ had rheumatoid arthritis (Figure 1). 154 patients (60.6\%) underwent unilateral knee replacement and 100 patients underwent bilateral knee replacement, out of which $18.1 \%$ had single stage and $21.3 \%$ had staggered bilateral. All poly implant was used in $83.1 \%$ of patients and in $16.9 \%$ of patients metal backed was used (Table 2).

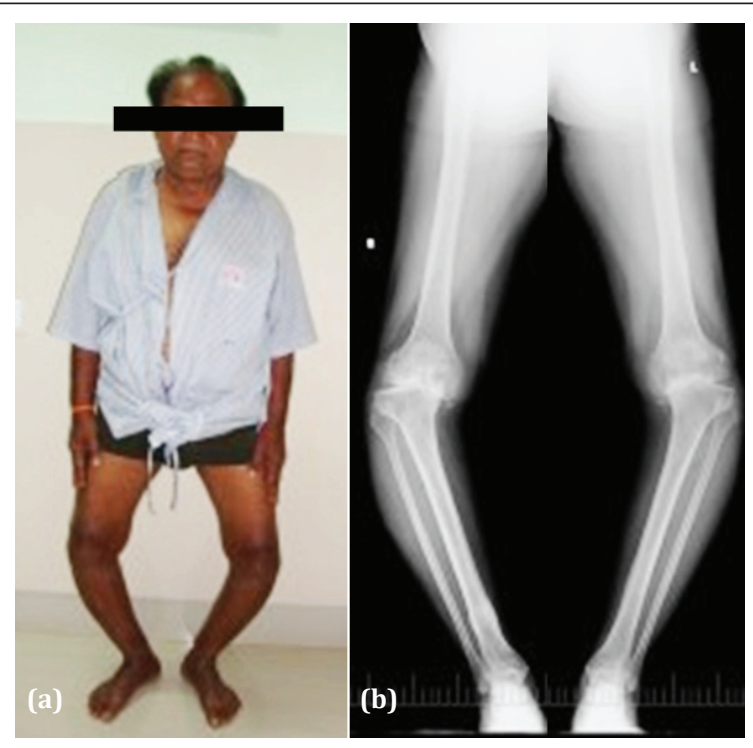

Figure 1a,b: Pre-operative clinical photograph and X-ray.

Table 2: Diagnosis related parameters.

\begin{tabular}{|lcc|}
\hline Parameters & Number & Percentage \\
\hline Pre-operative diagnosis & & \\
Rheumatoid arthritis & 48 & 18.9 \\
Osteoarthritis & 206 & 81.1 \\
Type of knee replacement & & \\
Unilateral & 154 & 60.6 \\
Bilateral single stage & 46 & 18.1 \\
Staggered bilateral & 54 & 21.3 \\
Type of implant & & \\
All polyethylene & 294 & 83.1 \\
Metal backed & 60 & 16.9 \\
\hline
\end{tabular}

Preoperatively there was no significant difference in the range of motion between various age groups. At 3 months ( $\mathrm{r}-0.04), 6$ months ( $\mathrm{r}-0.08)$, one year $(\mathrm{r}-0.04), 2$ year $(\mathrm{r}-0.27)$. There was no correlation between age and postoperative range of motion.

There was no significant preoperative difference in range of motion in both sex. Though the male had better range of motion at one year follow up ( $p$ 0.0465 ), at 2 year the difference was not statistically significant.

Patients with body mass index less than 25 had better range of motion at all the points of surgery 
when compared to patients with body mass index 25 to 30 and more than 30 group (preoperative p 0.032 , 3 months p 0.0082, 6 months p 0.027, one year $p$ $0.042,2$ years $p$ 0.0394).

Preoperatively there was a significant difference of range of motion when patients of rheumatoid arthritis were compared to those of osteoarthritis. Rheumatoid arthritis patients continued to have statistically significant decreased range of motion till 6 months after surgery (p0.0484). At one year and 2 year follow up this difference disappeared.

Patients who underwent bilateral knee replacement (Figure 2), either single stage or staggered had better range of motion at 3 months ( $\mathrm{p} 0.0291 \mathrm{r} 0.14$ ) and at 6 months ( $p 0.0128$ r 0.16), however this difference disappeared at one year ( $\mathrm{p} 0.585$ r 0.13 ) and at 2 year (p 0.515 r-0.06) (Table 3).

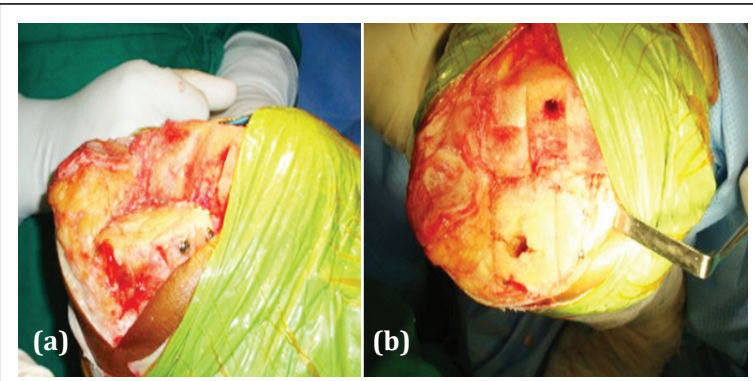

Figure 2a,b: Intra operative.

Table 3: Correlation of ROM with different parameters.

\begin{tabular}{|c|c|c|c|c|c|}
\hline \multirow{2}{*}{ Various factors } & \multicolumn{5}{|c|}{ ROM } \\
\hline & Pre operative & 3 months & 6 months & one year & 2 years \\
\hline \multicolumn{6}{|l|}{ Age } \\
\hline $50-59$ & $92.9 \pm 22.8$ & $101.0 \pm 9.3$ & $111.4 \pm 9.2$ & $116.9 \pm 8.2$ & $123.4 \pm 6.4$ \\
\hline $60-69$ & $92.9 \pm 19.5$ & $98.4 \pm 9.1$ & $109.3 \pm 9.0$ & $115.4 \pm 9.9$ & $119.4 \pm 8.8$ \\
\hline $70-80$ & $90.5 \pm 18.6$ & $98.4 \pm 9.1$ & $109.3 \pm 9.0$ & $115.9 \pm 12.3$ & $118 \pm 9.8$ \\
\hline r-value & -0.04 & -0.01 & -0.08 & -0.04 & -0.27 \\
\hline \multicolumn{6}{|l|}{ Sex } \\
\hline Male & $92.4 \pm 20.1$ & $99.2 \pm 9.8$ & $110 \pm 10.3$ & $117.4 \pm 11.8$ & $121.7 \pm 8.2$ \\
\hline Female & $92.4 \pm 20.4$ & $99.2 \pm 9.1$ & $109.9 \pm 9.5$ & $115 \pm 8.7$ & $120.3 \pm 8.2$ \\
\hline r-value & -0.01 & 0.01 & -0.01 & $-0.12^{*}$ & -0.08 \\
\hline \multicolumn{6}{|l|}{$B M I$} \\
\hline Up to 25 & $96 \pm 11.1$ & $104.1 \pm 9.1$ & $114.4 \pm 10.2$ & $119.9 \pm 9.2$ & $128.3 \pm 2.9$ \\
\hline $25.1-30$ & $92 \pm 20.4$ & $98.9 \pm 9.3$ & $109.6 \pm 9$ & $115.6 \pm 9.7$ & $123 \pm 8$ \\
\hline Above 30 & $88.8 \pm 9.1$ & $94.6 \pm 9.8$ & $105.9 \pm 10.3$ & $112 \pm 7.3$ & $115.8 \pm 7.6$ \\
\hline r-value & $-0.613^{*}$ & $-0.713^{* *}$ & $-0.653^{*}$ & $-0.513^{*}$ & $0.607^{*}$ \\
\hline \multicolumn{6}{|l|}{ Preoperative diagnosis } \\
\hline Rheumatoid Arthritis & $86.2 \pm 25$ & $96.4 \pm 8.3$ & $108.2 \pm 9.4$ & $113.7 \pm 9.8$ & $118.8 \pm 5.9$ \\
\hline Osteoarthritis & $93.8 \pm 18.8$ & $99.9 \pm 9.5$ & $110.3 \pm 9.8$ & $116.6 \pm 10$ & $121.4 \pm 8.6$ \\
\hline r-value & $0.15^{*}$ & $0.15^{* *}$ & 0.09 & 0.12 & 0.16 \\
\hline \multicolumn{6}{|c|}{ Type of knee replacement } \\
\hline Unilateral & $90 \pm 21.3$ & $97.5 \pm 10$ & $108 \pm 10.6$ & $114.5 \pm 11.1$ & $119.4 \pm 5.6$ \\
\hline Bilateral single stage & $94.1 \pm 18.8$ & $100.9 \pm 9.4$ & $111.5 \pm 9.3$ & $116.6 \pm 8$ & $123.6 \pm 7.5$ \\
\hline
\end{tabular}




\begin{tabular}{|c|c|c|c|c|c|}
\hline Staggered bilateral & $94.3 \pm 19.9$ & $100.5 \pm 7.9$ & $111.5 \pm 8.3$ & $117.5 \pm 9.5$ & $119.1 \pm 9.6$ \\
\hline r-value & 0.09 & 0.14 & $0.16^{*}$ & 0.13 & -0.06 \\
\hline \multicolumn{6}{|l|}{ Type of implant } \\
\hline All polyethylene & $91.7 \pm 20.5$ & $99.1 \pm 9.4$ & $109.9 \pm 9.6$ & $116.3 \pm 10.1$ & $120.9 \pm 8.4$ \\
\hline Metal backed & $95.5 \pm 19.3$ & $99.7 \pm 9.3$ & $110 \pm 10.3$ & $111.7 \pm 7.2$ & $118.5 \pm 4.9$ \\
\hline r-value & 0.07 & 0.02 & 0.003 & $-0.13^{*}$ & -0.09 \\
\hline
\end{tabular}

* - significant, ** - highly significant

The posterior tibial slope had no correlation with the postoperative range of motion at all the points of follow up. The fixed flexion deformity had a negative correlation with the postoperative range of motion preoperatively ( $\mathrm{r}-0.64)$, at 3 months $(\mathrm{r}-0.56)$, at 6 months(r-0.53), at one year ( $r-0.67)$. However at
2 years there was no correlation $(\mathrm{r}-0.45)$. Various deformity, valgus deformity, mediolateral and anteroposterior instability had no correlation with postoperative range of motion at all the points of follow up (Table 4) (Figures 3 and 4).

Table 4: Correlation coefficient of ROM with various factors.

\begin{tabular}{|c|c|c|c|c|c|}
\hline Factors & Pre operative & 3 months & 6 months & one year & 2 years \\
\hline Pre-operative range of motion & - & $0.7^{* *}$ & $0.68^{* *}$ & $0.71^{* *}$ & $0.61^{* *}$ \\
\hline Posterior tibial slope & -0.03 & -0.03 & -0.01 & 0.01 & 0.06 \\
\hline Fixed flexion deformity & $-0.64^{* *}$ & $-0.56^{* *}$ & $-0.53^{* *}$ & $-0.67^{* *}$ & -0.45 \\
\hline Varus deformity & -0.06 & -0.06 & -0.1 & -0.01 & 0.43 \\
\hline Valgus deformity & 0.28 & -0.26 & 0.07 & -0.36 & 0.16 \\
\hline $\begin{array}{l}\text { Mediolateral } \\
\text { instability }\end{array}$ & -0.15 & -0.1 & -0.12 & -0.2 & 0.11 \\
\hline $\begin{array}{l}\text { Antero posterior } \\
\text { instability }\end{array}$ & 0.02 & -0.09 & -0.07 & 0.01 & -0.09 \\
\hline
\end{tabular}

* - significant, ** - highly significant

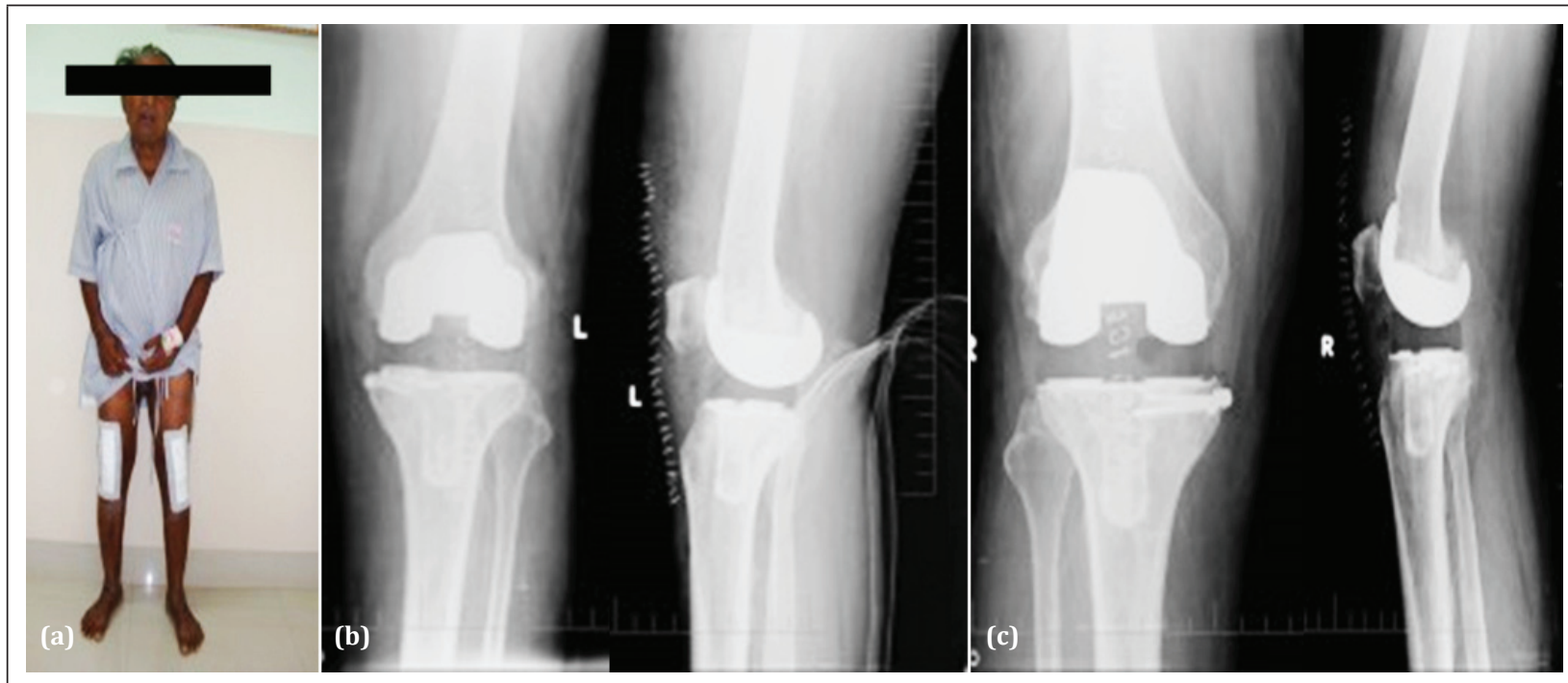

Figure 3a-c: Post-operative clinical photograph and X-ray - left, right knee. 
The preoperative range of motion had a statistical correlation with the postoperative range of motion at all the points of follow up and in all the age groups, $\mathrm{p}<0.0001$ (Table 5).

Table 5: Comparison of pre and post operative range of motion according to age.

\begin{tabular}{|lccccccc|}
\hline \multirow{2}{*}{ Age in years } & Pre-operative & \multicolumn{5}{c|}{ Follow up } & p-value \\
\cline { 3 - 7 } & & 3 months & 6 months & one year & 2 year & \\
\hline $50-59$ & $92.9 \pm 22.8$ & $101.0 \pm 9.3$ & $111.4 \pm 9.2$ & $116.9 \pm 8.2$ & $123.4 \pm 6.4$ & $<0.0001$ \\
$60-69$ & $92.9 \pm 19.5$ & $98.4 \pm 9.1$ & $109.3+\_9.0$ & $115.4 \pm 9.9$ & $119.4 \pm 8.8$ & $<0.0001$ \\
$70-80$ & $90.5 \pm 18.6$ & $98.4 \pm 9.1$ & $109.3 \pm 9.0$ & $115.9 \pm 12.3$ & $118 \pm 9.8$ & $<0.0001$ \\
\hline
\end{tabular}

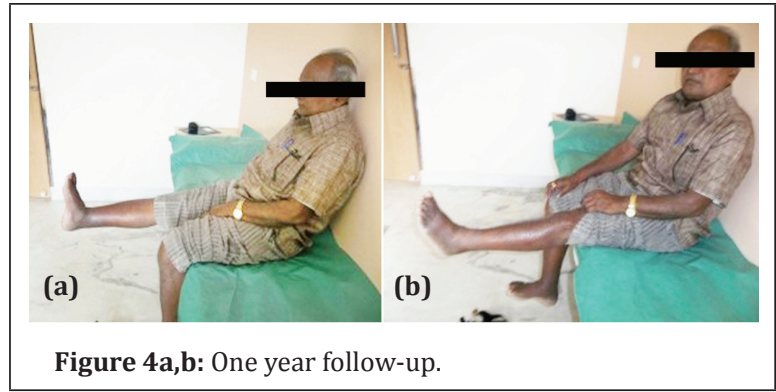

\section{Discussion}

Improved range of motion is an important goal after total knee arthroplasty as it is an important determinant of patient satisfaction. Activities of daily living like deep knee bend require a knee flexion of 110 degree or more, if this can be achieved improvement in the performances of activities can be expected.

A number of previous studies have shown preoperative range of motion as one of the most important factors predicting postoperative range of motion [8-12], however controversy exists regarding other factors like age, sex, body mass index, posterior cruciate ligament retention, ligament balancing and postoperative physiotherapy. The purpose of this study was to evaluate these factors in Indian patients using a single contemporary knee arthroplasty design.

The influence of age on postoperative knee range of motion remains controversial. Horikawa et al found that preoperative range of motion was not correlated with age, but that there was a weak correlation between postoperative range of motion and age $(r=0.277, P$ 0. 05) [13]. Schurman et al. divided 25 patients with preoperative range of motion of less than 78 degree into 2 groups: one group of patients 62 years or younger and a second group older than 63 years. The younger group showed a mean postoperative range of motion of 83 degree, whereas the older group had a mean value of 100 degree, demonstrating that the age was a factor [14]. In contrast, Harvey et al and Anouchi et al reported no correlation between age and postoperative knee range of motion $[15,16]$.

In the present study it was observed that men had better range of motion at one year follow up but however this difference was not significant at 2 years follow up. Our study agrees with the report of Schurman et al., that sex did not appear to be an important factor affecting knee joint range of motion [17]. Further, Shuklka also reported that, no significant correlation between sex and range of motion post operatively [18].

In our study patients with low body mass index less than 25 had better range of movements at all the points of follow up and was statistically significant. There are some reports indicating that obesity has an adverse effect on postoperative knee range of motion due to soft tissue impingement between the femur and the tibia, which restricts flexion of the knee $[10,19]$, however, a higher body mass index alone does not explain the distribution of adipose tissue [20]. It is well known that the pattern of adipose tissue distribution varies between men and women. Men tend to have a more "android" fat distribution presenting with adipose tissue in the central or abdominal region, whereas women demonstrate a "gynoid" lower body or gluteal-femoral deposition. Shoji et al concluded that obese patients accounted for a larger percentage of the patients with a poor range of motion [19]. Lizaur et al. reported that body mass index was significantly correlated $(r=0.25, P=$ 0.023 ) with postoperative range of motion [10]. Our study findings similar with Shoji et al and Lizaur et 
al., body mass index had a strong correlation with postoperative range of motion. In another study reported, patients with lower BMI (less than 30 ) had significantly higher flexion attained against ones of higher BMI (higher than 30) [18].

Regarding, preoperative range of motion, most reports have demonstrated that a greater postoperative flexion was achieved in patients with greater preoperative range of motion of the knee joint. Kurosaka et al. and Shukla $\mathrm{H}$ et al. reported that preoperative range of motion of the knee joint was the most important factor [18, 21]. A similar result also reported by our study results has clearly demonstrated positive correlation between preoperative flexion and postoperative flexion at all the points of follow up with a statistically significant relationship irrespective of the age group.

There was no study report available in the literature regarding the relationship between postoperative flexion and whether the patient had unilateral, bilateral single staged or staggered bilateral knee replacement. We found better range of motion in patients with bilateral knee replacement either single stage or staggered as compared to unilateral at 3 months and 6 months. This may be attributed to the fact that patients who underwent bilateral knee replacement probably had a prolonged hospital stay and better rehabilitation process as compared to unilateral knee replacements. But, however there was no difference between these groups at one year and 2 year follow ups.

In our study, preoperative tibiofemoral deformity was not found to be a significant factor affecting postoperative range of motion. Similar findings were reported by Kawamura and Bourne [22] and Shurman et al. [14]. However fixed flexion deformity had a negative correlation with preoperative and postoperative follow up till one year. However, at 2 years it was not statistically significant.

In a comparison of metal-backed and all-polyethylene tibial components of the PFC knee, Rodriguez et al and Dalury et al found that the clinical, functional and radiographic results of the implants were equivalent $[23,24]$. Udomkiat et al. in their study found that the mean flexion for allpolyethylene tibial knees was $120.5^{\circ} \pm 8.0^{\circ}$ and for metal backed tibial knees was $118.3^{\circ} \pm 10.4^{\circ}(\mathrm{P}=0.300)[25]$. We had better range of motion in all polyethylene group at one year follow up ( $\mathrm{p}=0.033)$, but however there was no statistical difference at 2 years between the both groups. We agree with Rodriguez et al and Udomkiat et al [23, 25] that the results of implants were equivalent in both groups.

Some authors have hypothesized that proximal tibial slope influences postoperative ROM. Walker and Garg, in a computer modelling study, attempted to determine the effect of proximal tibial slope on postoperative ROM. The effects of a 10 degree posterior tilt, neutral tilt, and a 10 degree anterior tilt were compared. He concluded that a 10 degree posterior tilt produced no less than 30 degree of additional flexion when compared with the neutral tilt and anterior tilt had the opposite effect. Although one could expect these results in a computer simulation, the model may have overlooked some very important anatomical and physiological variables. Clearly, the in vivo situation varies significantly from the analytical computer modeling because of confounding variables. In our study we did not find any correlation between the posterior tibial slope and postoperative range of motion [26].

It was understood that, there is no study report available in the literature regarding the relationship between knee scores and age, sex, body mass index, diagnosis, type of knee replacement, implants involved and deformities. We found patients with low body mass index had better knee scores at all the points of follow up and also patients with osteoarthritis had better knee score preoperatively and postoperatively at 3 months. We found no correlation between knee score and varus, valgus, fixed flexion deformities and instabilities. In another study reported, there was moderate positive correlation between lateral joint laxity and the standard activity score in extension [27].

Whereas in another study, Oxford knee score and Western Ontario and McMaster Universities Osteoarthritis Index ((WOMAC) score were used for function measure and found that, average Oxford Knee Score was 43 and WOMAC 91. Function of tibial components (80\%), knees (31\%) and limbs (7\%) that were aligned in varus was similar to patients aligned in-range [28].

\section{Conclusion}

Patients with decreased preoperative range of 
motion have to be properly counselled regarding their expectation for a better postoperative range of motion. A more keen watch has to be kept in all young female patients with early onset of arthritis and mange them aggressively with conservative therapy. Both all polyethylene and metal backed group fared equally well, but however the number of people who were replaced with metal backed implants were significantly less, hence a larger study with a longer follow up is the need of the hour to evaluate the survivorship of the prosthesis.

\section{Limitations}

A more comprehensive validatory study is required to compare the efficacy of various knee scores in evaluation of functional outcomes of the patients undergoing total knee arthroplasty.

As our study had a maximum follow up of only 2 years, it is recommended that the same patients be followed up for a longer periods to ascertain the fate of various variables evaluated here.

\section{Conflicts of interest}

Authors declare no conflicts of interest.

\section{References}

[1] Shetty AA, Tindall A, Ting P, Heatley FW. The evolution of total knee arthroplasty. Part1: Introduction and first steps. Current Orthopaedics. 2003; 17(4):322-325.

[2] Kansara D, Markel DC. The effect of posterior tibial slope on range of motion after total knee arthroplasty. J Arthroplasty. 2006; 21(6):809-813.

[3] Insall JN, Ranawat CS, Aglietti P, Shine J. A comparison of four models of total knee-replacement prostheses. J Bone Joint Surg Am. 1976; 58(6):754-765.

[4] Shetty AA, Tindall A, Ting P, Heatley FW. The evolution of total knee arthroplasty. Part II: The hinged knee replacement and the semi-constrained knee replacement. Current Orthopaedics. 2003; 17(5):403-407.

[5] Yau WP, Chiu KY, Tang WM, Ng TP. Residual posterior femoral condyle osteophyte affects the flexion range after total knee replacement. Int Orthop. 2005; 29(6):375-379.

[6] Kotani A, Yonekura A, Bourne RB. Factors influencing range of motion after contemporary total knee arthroplasty. J Arthroplasty. 2005; 20(7):850-856.

[7] Insall JN, Dorr LD, Scott RD, Scott WN. Rationale of the knee society clinical rating system. Clin Orthop Relat Res. 1989; 248:13-14

[8] Gatha NM, Clarke HD, Fuchs R. Factors affecting postoperative range of motion after total knee arthroplasty. J Knee Surg. 2004; 17(4):196-202.

[9] Thompson NW, Mockford BJ, Beverland DE. Factors influencing range of motion following total knee arthroplasty. J Bone Joint Surg Br. 2005; 87(Suppl II):156.
[10] Lizaur A, Marco L, Cebrian R. Preoperative factors influencing the range of movement after total knee arthroplasty for severe osteoarthritis. J Bone Joint Surg Br. 1997; 79(4):626629.

[11] Ritter MA, Harty LD, Davis KE. Predicting range of motion after total knee arthroplasty: clustering, log-linear regression, and regression tree analysis. J Bone Joint Surg Am. 2003; 85(7):1278-1285.

[12] Schurman DJ, Roger DE. Total knee arthroplasty: range of motion across five systems. Clin Orthop Relat Res. 2005; 430:132-137.

[13] Horikawa K, Wakabayashi H, Uchida A. Factors influencing the postoperative range of motion after total knee arthroplasty. J Chubu Seisai. 1999:717.

[14] Schurman DJ, Matityahu A, Goodman SB, Maloney W, Woolson S, et al. Prediction of postoperative knee flexion in Insall- Burstein II total knee arthroplasty. Clin Orthop. 1998; 353:175-184.

[15] Harvey IA, Barry K, Kirby SPJ. Factors affecting the range of movement of total knee arthroplasty.J Bone Joint Surg Br. 1993; 75(6):950-955.

[16] Anouchi YS, McShane M, Kelly. Range of motion in total knee replacement. Clin Orthop Relat Res. 1996; 331:87-92.

[17] Schurman DJ, Parker JN, Ornstein D. Total condylar knee replacement. J Bone Joint Surg Am. 1985; 67(7):10061014.

[18] Shukla H, Goregaonkar A, Manghwani J, Rajesh S, Naskar R. Factors determing range of motion post operatively in total knee arthroplasty. Indian J Orthop Surg. 2018; 4(3):245248.

[19] Shoji H, Solomonow M, Yoshino S. Factors affecting postoperative flexion in total knee arthroplasty. Orthopedics. 1990; 13(6):643-649.

[20] Gadinsky NE, Ehrhardt JE, Urband C, Westrich GH. Effect of body mass index on range of motion and manipulation after total knee arthroplasty. J Arthroplasty. 2011; 26(8):11941197.

[21] Kurosaka M, Yoshiya S, Mizuno K. Maximizing flexion after total knee arthroplasty. J Arthroplasty.2002;17(4Supp1):5962.

[22] Kawamura H, Bourne RB. Factors affecting range of flexion after total knee arthroplasty. J Orthop Sci. 2001; 6(3):248252.

[23] Rodriguez JA, Baez N, Rasquinha V. Metalbacked and allpolyethylene tibial components in total knee replacement. Clin Orthop Relat Res. 2001; 392:174-183.

[24] Dalury DF, Pomeroy DL. Midterm Results of all-polyethylene tibial components in primary total knee arthroplasty. J Arthroplasty. 2009; 24(4):620-625.

[25] Udomkiat P, Dorr LD, Long W. Matched-pair analysis of all-polyethylene versus metal-backed tibial components. J Arthroplasty. 2001; 16(6):689-695.

[26] Walker PS, Garg A. Range of motion in total knee arthroplasty. A computer analysis. Clin Orthop Relat Res. 1991; 262:227235.

[27] Tsukiyama H, Kuriyama S, Kobayashi M, Nakamura S, Furu $\mathrm{M}$, et al. Medial rather than lateral knee instability correlates with inferior patient satisfaction and knee function after total knee arthroplasty. Knee. 2017; 24(6):1478-1484.

[28] Howell SM, Papadopoulos S, Kuznik K, Ghaly LR, Hull ML. Does varus alignment adversely affect implant survival and function six years after kinematically aligned total knee arthroplasty? Int Orthop. 2015; 39(11):2117-2124. 